\title{
Entlassung am nächsten Tag bei Einweisung nach Suizidankündigung - Kontra
}

\section{Discharge on the Day After Admission Following Announcement of Suicide - Contra}

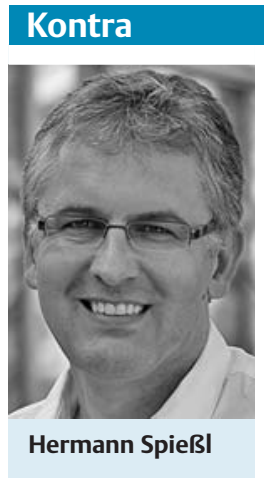

Regelmäßig ergibt sich neuerdings in psychiatrischen Kliniken folgende Situation: Sie/er wurde von ihrem/seiner Freund/in verlassen und teilt per SMS mit, dass sie/er nicht mehr leben wolle. Die vom SMS-Empfänger aufgeregt herbeigerufene Polizei bringt sie/ihn in die Klinik. Gleich im Aufnahmegespräch teilt sie/er mit, dass die SMS nicht ernst gemeint gewesen sei und sie/er nach Hause möchte. Wie ist nun durch den Arzt zu entscheiden?

Aus medizinischer und juristischer Sicht ist zu prüfen, ob eine akute Suizidalität vorliegt. Eine solche Prüfung ist notwendig, damit dem Arzt kein fahrlässiges Handeln bzw. eine Unterlassung seiner ärztlichen Sorgfaltspflichten vorgeworfen werden kann. Es ist - auch für einen Richter im Falle eines nachfolgenden Suizidversuchs oder Suizides - offensichtlich, dass eine hinreichende Diagnostik der Suizidalität nicht allein in einem einzigen Gespräch mit dem Patienten möglich ist. Im schlimmsten Fall drohen zivilrechtlich Schadensersatzansprüche wegen Nichtverhinderung einer suizidalen Handlung und strafrechtlich der Vorwurf einer fahrlässigen Tötung durch Unterlassung. Gerade bei stationärer Behandlung haben Ärzte eine sog. Garantenpflicht, da von juristischer Seite kaum eine Eigenverantwortlichkeit des Suizidenten gesehen wird. Die aus Patientensicht sehr wichtige Autonomie und die gesellschaftspolitisch (und juristisch) hoch gewertete Selbstbestimmung des Patienten müssen hier aus meiner Sicht eine Einschränkung erfahren, sodass ggf. eine Unterbringung beantragt werden muss.

Solche Suizidankündigungen von meist jungen Erwachsenen sind grundsätzlich ernst zu nehmen, da bei fast $90 \%$ der
Adoleszenten mit Suizidideen und bei über 95\% der Adoleszenten mit Suizidversuchen eine psychische Störung vorliegt [1]. Die Mehrzahl der Heranwachsenden war bislang noch nicht in Behandlung [2]. 45\% der Adoleszenten haben drei Monate nach einem Suizidversuch anhaltend Suizidgedanken, $12 \%$ unternehmen einen erneuten Suizidversuch [3]. Grundsätzlich gelten Menschen mit psychischen Erkrankungen, in belastenden Lebenssituationen und mit früherer oder aktueller Nähe zu Suizidalität bekanntermaßen als Hochrisikogruppen für Suizide. Nicht selten liegt auch eine Kombination dieser Faktoren (und anderer Risikofaktoren) vor.

Der erste Schritt ist somit eine hinreichende Diagnostik. Die Abklärung von Suizidalität (unter Berücksichtigung bekannter Risikofaktoren) bezieht sich auf die aktuelle Symptomatik, mögliche zugrunde liegende psychische Störungen, die aktuelle psychosoziale Situation, zugrunde liegende Persönlichkeitsfaktoren und auch die Familiengeschichte. Sie beinhaltet zudem immer spezifische Fragen zur Suizidalität, d.h. nach Suizidgedanken, konkreten Ideen, Vorbereitungen, früheren Versuchen und dem aktuellen Handlungsdruck, aber auch nach früherer Bewältigung, haltenden Faktoren und Zukunftsperspektiven. Eine Fremdanamnese ist zudem oft unumgänglich. Eine solche umfassende Abklärung ist kaum bis zum nächsten Tag nach der Einweisung zu schaffen, insbesondere wenn diese erst nachts zuvor erfolgte.

Klar ist die Einschätzung z.B. bei einem depressiven oder schizophrenen Patienten, der sich nicht von Suizidideen bzw. vom Suizidversuch distanziert, drängende Suizidgedanken erlebt und hoffnungslos ist. Diese Patienten bleiben meist auch freiwillig in stationärer Behandlung. Wenig schwierig ist in der Regel auch die Einschätzung eines Patienten, der im alkoholisierten Zustand eine Suizidäußerung macht und sich - wenn wieder nüchtern - glaubhaft und nachvollziehbar distanziert. Im intoxikierten Zustand ist auf- grund der reduzierten Impulskontrolle ein Verbleiben in der Klinik erforderlich, nach Ausnüchterung und nach abgeschlossener hinreichender Diagnostik (s.o.) erfolgt die Entlassung - oder ggf. die freiwillige Weiterbehandlung seiner Suchterkrankung.

Problematischer ist die Einschätzung bei Patienten mit noch unklarer psychischer Störung oder mit (fraglichen) Persönlichkeitsstörungen. Hier besteht in unserer Klinik die Regel, dass keine Entlassung am nächsten Tag bei Einweisung nach Suizidankündigung erfolgt, insbesondere, wenn einer oder mehrere folgender Punkte vorliegen: Der Patient reagiert gereizt, wirkt eingeengt, zieht sich zurück, zeigt mangelnde Impulskontrolle, ist intoxikiert, hat aktuellen zur Suizidalität führenden Konflikt nicht gelöst, kann wenig konkret darstellen, was sich an seiner Einstellung und seiner (möglicherweise desolaten) sozialen Situation geändert hat, hat einen/mehrere Suizidversuche bzw. suizidale Krisen in der Vorgeschichte, spricht nicht offen über seine Suizidalität oder spielt seinen Suizidversuch herunter bzw. stellt ihn als „unbeabsichtigt“ dar. Auch eine Suizidalität mit zunehmend (!) instrumentellem Charakter spricht gegen eine sofortige Entlassung. In diesem $\mathrm{Zu}$ sammenhang wäre auch die Frage zu diskutieren, wie hoch die Eigenverantwortlichkeit in solchen Situationen bei Borderline-Patienten ist, wenn man die bekannten strukturellen und funktionellen Veränderungen in zentralen, frontal-limbischen und kortikalen Regelkreisen bei BPS bedenkt. Es geht nicht um Hospitalisierung, sondern nur um die Nichtentlassung am nächsten Tag, wenn noch keine wirkliche Auseinandersetzung mit dem Vorfall, i.e. dysfunktionalen Verhalten, stattgefunden hat. Wir haben hier gute Erfahrungen mit ein paar Tagen Aufenthalt gemacht, um gemeinsam zu reflektieren und zu klären, z.B. auch mit Verhaltensanalysen (DBT). Eine zweiwöchige spezifische stationäre Behandlung zeigte sich in einer quasiexperimentellen Studie 
erfolgreicher im Vergleich zur Nichtbehandlung bezüglich Wiederholungsrisiko eines Suizidversuchs (5,6 vs. $15,4 \%$ ) und anderer psychopathologischer Variablen [4].

Einen Behandlungsfehler könnte es - neben einer nicht hinreichenden Exploration der Suizidalität (s.o.) - darstellen, hier Bagatellisierungstendenzen des Patienten mitzumachen und keine adäquate Therapie i.e. Krisenintervention einzuleiten, die folgende 3 Bereiche beinhaltet: 1. Beziehungsaufbau und Fürsorge für den Betroffenen, 2. Diagnostik der psychischen Erkrankung und Klärung der psychosozialen Situation, der Beziehungsstruktur und des Verhaltensrepertoires, 3. Weichenstellung, Therapie der psychischen Erkrankung und Motivation zur Nachsorge. Entscheidend - und nicht in einem einzigen Gespräch machbar - ist die Bearbeitung des gescheiterten Bewältigungsversuchs, die gemeinsame Erar- beitung alternativer Lösungen, die Verbesserung der Copingstrategien (für zukünftige Krisen) und das Wiederherstellen wichtiger Beziehungen. Dies - und am besten im Rahmen einer auf das suizidale Verhalten ausgerichteten kognitiven Verhaltenstherapie - ist dann auch suizidpräventiv wirksam [5].

\section{Literatur}

1 Nock MK, Green JG, Hwang I et al. Prevalence, correlates, and treatment of lifetime suicidal behavior among adolescents: results from the National Comorbidity Survey Replication Adolescent Supplement. JAMA Psychiatry 2013; 70: 300-310

2 Husky MM, Olfson M, He JP et al. Twelvemonth suicidal symptoms and use of services among adolescents: results from the National Comorbidity Survey. Psychiatr Serv 2012; 63: 989-996

3 Spirito A, Valeri S, Boergers J et al. Predictors of continued suicidal behavior in adolescents following a suicide attempt. J Clin Child Adolesc Psychol 2003; 32: 284 - 289
4 Nordentoft M, Branner J, Drejer K et al. Effect of a Suicide Prevention Centre for young people with suicidal behaviour in Copenhagen. Eur Psychiatry 2005; 20: 121 - 128

5 Tarrier N, Taylor K, Gooding P. Cognitivebehavioral interventions to reduce suicide behavior: a systematic review and meta-analysis. Behav Modif 2008; 32: 77-108

Sie haben eine eigene Meinung zu diesem Thema? Dann schreiben Sie uns an: psychiat-praxis@thieme.de!

Korrespondenzadresse Prof. Dr. med. Hermann SpießI

Klinik für Psychiatrie, Psychotherapie und Psychosomatik, Bezirkskrankenhaus Landshut Prof.-Buchner-Straße 22 84034 Landshut

h.spiess!@bkh-landshut.de

Bibliografie

DOI http://dx.doi.org/

10.1055/s-0035-1552734

Psychiat Prax 2015; 42: 357-358

(c) Georg Thieme Verlag KG

Stuttgart · New York

ISSN 0303-4259 